\title{
SOME REMARKS ON THE DAUGAVET EQUATION
}

\author{
P. WOJTASZCZYK
}

(Communicated by William J. Davis)

\begin{abstract}
We show that direct sums in $l_{1}$ and $l_{\infty}$ sense of Banach spaces that satisfy the Daugavet Equation (1) also satisfy it. We also show that most uniform algebras satisfy (1) for weakly compact operators.
\end{abstract}

\section{INTRODUCTION}

In his 1963 paper [D], Daugavet observed that for each linear compact operator $T: C[0,1] \rightarrow C[0,1]$ one has

$$
\|I+T\|=1+\|T\| .
$$

This was extended later to weakly compact operators. We will say that a Banach space $X$ satisfies the Daugavet Equation (DE for short) for some class of operators if for every operator $T: X \rightarrow X$ from this class (1) holds. The general history of the subject is given in $[\mathrm{A}]$. This paper got me interested in the problem. Before $[\mathrm{A}]$ the only known spaces satisfying (DE) for weakly compact operators were atomless $L_{1}(\mu)$ and $C(K)$ spaces. The main motivation of [A] was to extend this class. We solve the problem asked in [A] that finite $l_{1}$ and $l_{\infty}$ sums of spaces with (DE) for weakly compact operators satisfy (DE) for weakly compact operators, thus recovering and extending results from [A]. Actually the result holds when weakly compact operators are replaced by any operator ideal. Next we greatly extend the class of spaces satisfying (DE) for weakly compact operators by showing that "most" uniform algebras satisfy (DE) for weakly compact operators. This result has many interesting consequences, e.g., there are spaces satisfying (DE) for weakly compact operators that contain 1-complemented, infinite-dimensional, reflexive subspaces. Clearly no infinitedimensional, reflexive subspace can satisfy (DE) for weakly compact operators. We extend it a bit by showing that no space with the Radon-Nikodym property (cf. [DU]) can have (DE) for one-dimensional operators.

Our notation and terminology is standard. All unexplained results and notions can be found in [W].

\section{RESULTS}

Exposed points. We start with the following observation.

Received by the editors January 27, 1991.

1991 Mathematics Subject Classification. Primary 46B04; Secondary 46J10, 47B07. 
Proposition 1. If $X$ is a Banach space that satisfies the Daugavet Equation for one-dimensional operators, then $B_{X}$, the closed unit ball of $X$, does not have a strongly exposed point.

Proof. Suppose that $x_{0} \in B_{X}$ is a strongly exposed point. Then there exists a functional $x^{*} \in X^{*}$ with $x^{*}\left(x_{0}\right)=1=\left\|x^{*}\right\|$ such that if $\left\|x_{n}\right\| \leq 1$ and $x^{*}\left(x_{n}\right) \rightarrow 1$ then $x_{n} \rightarrow x_{0}$. Let us consider an operator $T(x)=x^{*}(x) h$ for $h \in \operatorname{ker} x^{*}$. We have $\|I+T\|=1+\|T\|=1+\|h\|$. Thus there exists a sequence $\left(x_{n}\right)_{n=1}^{\infty}$ in $B_{X}$ such that $\left\|x_{n}+x^{*}\left(x_{n}\right) h\right\| \rightarrow 1+\|h\|$. This implies that $\left|x^{*}\left(x_{n}\right)\right| \rightarrow 1$. We can assume $x^{*}\left(x_{n}\right) \rightarrow 1$ so $x_{n} \rightarrow x_{0}$. This gives $\left\|x_{0}+h\right\|=1+\|h\|=\left\|x_{0}\right\|+\|h\|$ for every $h \in \operatorname{ker} x^{*}$, so $X \cong\left(\operatorname{ker} x^{*} \oplus \lambda x_{0}\right)_{1}$. But then for $P(x)=x^{*}(x) x_{0}$ we have $\|I-P\|=1$, so $X$ does not satisfy (DE) for one-dimensional operators.

Corollary 1. If $X$ has the Radon-Nikodym property, then $X$ does not satisfy $(D E)$ for one-dimensional operators.

Proof. By Phelps's Theorem (see [DU, VII, Theorem 3]), spaces with the RadonNikodym property have strongly exposed points.

As a particular example let us mention that $H_{1}(D)$ does not satisfy (DE).

Corollary 2. Every Banach space can be renormed to fail (DE).

Proof. It is enough to introduce a strongly exposed point to the unit sphere in the new norm. For $x_{0} \in X$ with $\left\|x_{0}\right\|>1$ the new ball can be given as $\overline{\operatorname{conv}}\left\{x_{0}, B_{X}\right\}$.

Additivity. The following theorem expresses a certain stability property of spaces satisfying (DE). It also solves the main problem left open in [A].

Theorem 1. If $\left(X_{j}\right)_{j=1}^{n}$ satisfy $(D E)$ for weakly compact operators where $n=$ $1,2, \ldots$ or $n=\infty$, then $\left(\sum_{j=1}^{n} X_{j}\right)_{1}$ and $\left(\sum_{j=1}^{n} X_{j}\right)_{\infty}$ satisfy $(D E)$ for weakly compact operators.

Proof. Let us consider the case $\left(\sum_{j=1}^{n} X_{j}\right)_{\infty}$ first. If $T:\left(\sum_{j=1}^{n} X_{j}\right)_{\infty} \rightarrow$ $\left(\sum_{j=1}^{n} X_{j}\right)_{\infty}$ is an operator, then we can identify $T$ with the sequence of operators $\left(T_{j}\right)_{j=1}^{n}$ where $T_{i}:\left(\sum_{j=1}^{n} X_{j}\right) \rightarrow X_{i}$ and clearly $\|T\|=\sup _{i}\left(\left\|T_{i}\right\|\right)$. If $T$ is weakly compact, then $T_{i}$ 's also are. To fix our attention let us assume that $\|T\|<\left\|T_{1}\right\|+\varepsilon$ and denote $\left(\sum_{j=2}^{n} X_{j}\right)_{\infty}$ by $Y$. Let us fix $\left(\bar{x}_{1}, \bar{x}_{2}\right) \in X_{1} \oplus Y$ such that $\left\|\bar{x}_{1}\right\|=\left\|\bar{x}_{2}\right\|=1$ and $\left\|T_{1}\left(\bar{x}_{1}, \bar{x}_{2}\right)\right\| \geq\left\|T_{1}\right\|-\varepsilon$. This is easily seen to be possible (cf. [A, Corollary to Lemma 5]). We define an operator $S: X_{1} \rightarrow X_{1}$ by the formula

$$
S\left(x_{1}\right)=T_{1}\left(x_{1}, 0\right)+x_{1}^{*}\left(x_{1}\right) T_{1}\left(0, \bar{x}_{2}\right)
$$

where $x_{1}^{*} \in X_{1}^{*}$ is such that $x_{1}^{*}\left(\bar{x}_{1}\right)=1=\left\|x_{1}^{*}\right\|$. Clearly $S$ is weakly compact. We have

$$
\left\|T_{1}\right\| \geq\|S\| \geq\left\|T_{1}\right\|-\varepsilon
$$

Indeed

$$
\begin{aligned}
\|S\| & =\sup _{\left\|x_{1}\right\| \leq 1}\left\|T_{1}\left(x_{1}, 0\right)+x_{1}^{*}\left(x_{1}\right) T_{1}\left(0, \bar{x}_{2}\right)\right\| \\
& \leq \sup _{\left\|x_{1}\right\| \leq 1,|\lambda| \leq 1}\left\|T_{1}\left(x_{1}, \lambda \bar{x}_{2}\right)\right\| \\
& \leq \sup _{\left\|x_{1}\right\| \leq 1,\left\|x_{2}\right\| \leq 1}\left\|T_{1}\left(x_{1}, x_{2}\right)\right\|=\left\|T_{1}\right\|
\end{aligned}
$$


and

$$
\left\|S\left(\bar{x}_{1}\right)\right\|=\left\|T_{1}\left(\bar{x}_{1}, 0\right)+T_{1}\left(0, \bar{x}_{n}\right)\right\|=\left\|T_{1}\left(\bar{x}_{1}, \bar{x}_{2}\right)\right\|=\left\|T_{1}\right\|-\varepsilon .
$$

Let us now consider the operator $I+S: X_{1} \rightarrow X_{1}$. Since $X_{1}$ satisfies (DE) for weakly compact operators, we can find $\tilde{x}_{1} \in X_{1}$ such that $\left\|\tilde{x}_{1}\right\|=1$ and $1+\|S\| \leq\left\|\tilde{x}_{1}+S\left(\tilde{x}_{1}\right)\right\|+\varepsilon$. Now we have

$$
\begin{aligned}
\|I+T\| & \geq\left\|(I+T)\left(\tilde{x}_{1}, x_{1}^{*}\left(\tilde{x}_{1}\right) \bar{x}_{2}\right)\right\| \geq\left\|\tilde{x}_{1}+T_{1}\left(\tilde{x}_{1}, x_{1}^{*}\left(\tilde{x}_{1}\right) \bar{x}_{2}\right)\right\| \\
& =\left\|\tilde{x}_{1}+T_{1}\left(\tilde{x}_{1}, 0\right)+x_{1}^{*}\left(\tilde{x}_{1}\right) T_{1}\left(\bar{x}_{2}\right)\right\|=\left\|\tilde{x}_{1}+S\left(\tilde{x}_{1}\right)\right\| \\
& \geq 1+\|S\|-\varepsilon \geq 1+\left\|T_{1}\right\|-2 \varepsilon=1+\|T\|-3 \varepsilon .
\end{aligned}
$$

Since $\varepsilon$ was arbitrary and obviously $\|I+T\| \leq 1+\|T\|$, we get the claim.

The argument for the case $\left(\sum_{j=1}^{n} X_{j}\right)_{1}$ is basically the dual version of the above. Given $T:\left(\sum_{j=1}^{n} X_{j}\right)_{1} \rightarrow\left(\sum_{j=1}^{n} X_{j}\right)_{1}$, we write it as $\left(T_{i}\right)_{i=1}^{n}$ where $T_{i}: X_{i} \rightarrow\left(\sum_{j=1}^{n} X_{j}\right)_{1}$. We have $\|T\|=\sup _{i}\left(\left\|T_{i}\right\|\right)$. To fix the notation let us assume that $\|T\|<\left\|T_{1}\right\|+\varepsilon$. We write $T_{1}\left(x_{1}\right)=\left(\alpha\left(x_{1}\right), \beta\left(x_{1}\right)\right)$ where $\alpha: X_{1} \rightarrow X_{1}$ and $\beta: X_{1} \rightarrow Y$ where $Y=\left(\sum_{j=2}^{n} X_{j}\right)_{1}$. Let us fix $\bar{x}_{1} \in X_{1}$ such that $\left\|\bar{x}_{1}\right\|=1$ and $\left\|T_{1}\left(\bar{x}_{1}\right)\right\|=\left\|\alpha\left(\bar{x}_{1}\right)\right\|+\left\|\beta\left(\bar{x}_{1}\right)\right\| \geq\left\|T_{1}\right\|-\varepsilon$. Let us fix $x_{2}^{*} \in Y^{*}$ such that $\left\|x_{2}^{*}\right\|=1$ and $\left\|\beta\left(\bar{x}_{1}\right)\right\|=x_{2}^{*}\left(\beta\left(\bar{x}_{1}\right)\right)$. We put $x_{1}^{*}=\beta^{*}\left(x_{2}^{*}\right)$ and we define $S: X_{1} \rightarrow X_{1}$ by the formula $S\left(x_{1}\right)=\alpha\left(x_{1}\right)+x_{1}^{*}\left(x_{1}\right) v$ where $v=\alpha\left(\bar{x}_{1}\right) /\left\|\alpha\left(\bar{x}_{1}\right)\right\|$. If $T$ is weakly compact, then $S$ is also. For $x_{1} \in X_{1}$ we have

$$
\left\|S\left(\bar{x}_{1}\right)\right\| \leq\left\|\alpha\left(x_{1}\right)\right\|+\left|x_{1}^{*}\left(x_{1}\right)\right| \leq\left\|\alpha\left(x_{1}\right)\right\|+\left\|\beta\left(x_{1}\right)\right\| \leq\left\|T\left(x_{1}\right)\right\|
$$

and

$$
\begin{aligned}
\left\|S\left(\bar{x}_{1}\right)\right\| & =\left\|\alpha\left(\bar{x}_{1}\right)+x_{1}^{*}\left(\bar{x}_{1}\right) v\right\|=\left\|\alpha\left(\bar{x}_{1}\right)+\right\| \beta\left(\bar{x}_{1}\right)\|\cdot v\| \\
& =\left\|\alpha\left(\bar{x}_{1}\right)\right\|+\left\|\beta\left(\bar{x}_{1}\right)\right\|=\left\|T_{1}\left(\bar{x}_{1}\right)\right\| \geq\left\|T_{1}\right\|-\varepsilon .
\end{aligned}
$$

So $\left\|T_{1}\right\|-\varepsilon \leq\|S\| \leq\left\|T_{1}\right\|$. Since $X_{1}$ satisfies (DE) for weakly compact operators, there exists $z \in X_{1}$ such that $\|z\|=1$ and such that

$$
1+\|S\|=\|I+S\| \leq\|z+S(z)\|+\varepsilon=\left\|z+\alpha(z)+x_{1}^{*}(z) v\right\|+\varepsilon .
$$

Now we have

$$
\begin{aligned}
\|(I+T)(z, 0)\| & =\|z+\alpha(z)\|+\|\beta(z)\| \geq\|z+\alpha(z)\|+\left|x_{2}^{*}(\beta(z))\right| \\
& \geq\|z+\alpha(z)\|+\left|x_{1}^{*}(z)\right|=\|z+\alpha(z)\|+\left\|x_{1}^{*}(z) v\right\| \\
& \geq\left\|z+\alpha(z)+x_{1}^{*}(z) v\right\|=\|z+S(z)\| \\
& \geq 1+\|S\|-\varepsilon \geq 1+\left\|T_{1}\right\|-2 \varepsilon=1+\|T\|-3 \varepsilon .
\end{aligned}
$$

Remark. Observe that the only way the above argument uses weak compactness of an operator $T$ is to use the fact that weakly compact operators form an operator ideal. Thus if we consider the Daugavet Equation with respect to any other operator ideal (like compact operators or Dunford-Pettis operators) the additivity property expressed in the above theorem will also hold.

Uniform algebras. Now we consider (DE) for uniform algebras. Our general reference here is [G]. Suppose we have a uniform algebra, i.e., closed, separating points subalgebra $A \subset C(X)$ with $1 \in A$ and $X$ a compact space. A nonempty subset $K \subset X$ is called a peak set if there exists $f \in A$ such that $f \mid K=1$ and $|f(x)|<1$ for $x \in X \backslash K$. A generalised peak set is an intersection of peak sets. 
Proposition 2. Let $K \subset X$ be a generalised peak set. For $\phi \in A^{*}$ let $\mu_{\phi}$ be any norm-preserving extension of $\phi$ to a functional on $C(X)$ (i.e., a measure). We put $R_{K}(\phi)=\left(\mu_{\phi} \mid K\right) \mid A$ (i.e., we restrict the measure $\mu_{\phi}$ to the closed set $K$ and treat the restricted measure as a functional on $A$ ). Then $R_{K}$ is a well-defined, norm 1 projection in $A^{*}$.

Proof. It is basically a restatement of the Glicksberg Theorem on peak sets (see [G, II.12.7]) that says if $\mu \in A^{\perp}$ then $\mu \mid K \in A^{\perp}$. From this follows that if $\mu_{1}$ and $\mu_{2}$ are any two extensions of $\phi$ then $\mu_{1}-\mu_{2} \in A^{\perp}$, so $\left(\mu_{1} \mid K\right) \mid A=$ $\left(\mu_{2} \mid K\right) \mid A$. Thus $R_{K}$ is well defined. The rest is obvious.

Since for each $f \in A$ the function $|f|$ attains its maximum at some generalised peak point (i.e., one point generalised peak set) (cf. [G, II.12.10]), without loss of generality, we can assume that generalised peak points are dense in $X$, i.e., that we consider $A$ as an algebra on its Shilov boundary.

Proposition 3. Let $A \subset C(X)$ be a uniform algebra such that generalised peak points are dense in $X$ and $X$ has no isolated points. Let $U \subset X$ be a nonempty open set and let $T: A \rightarrow A$ be a weakly compact operator. For every $\varepsilon>0$ there exists a peak set $K \subset U$ such that $\left\|R_{K} T^{*}\right\|<\varepsilon$.

Proof. Let $\left(U_{k}\right)_{k=1}^{\infty}$ be a sequence of disjoint, open subsets of $U$. Since generalised peak points are dense in $X$, each $U_{k}$ contains a generalised peak point so (see [G, II.12.2]) there are peak sets $F_{k} \subset U_{k}$ for $k=1,2, \ldots$. Let us define an operator $P: A^{*} \rightarrow\left(\sum_{k=1}^{\infty} R_{F_{k}}\left(A^{*}\right)\right)_{1}$ by the formula $P(\phi)=\sum_{k=1}^{\infty} R_{F_{k}}(\phi)$. Since $\sum_{k=1}^{\infty}\left\|R_{F_{k}}(\phi)\right\| \leq\|\phi\|$ we see that $\|P\| \leq 1$. The operator $P T^{*}: A^{*} \rightarrow$ $\left(\sum_{k=1}^{\infty} R_{F_{k}}\left(A^{*}\right)\right)_{1}$ is weakly compact, so from the lemma below we infer that for given $\varepsilon>0$ there exists a $k$ such that $\left\|R_{F_{k}} T^{*}\right\|<\varepsilon$.

Lemma. Let $\left(Y_{n}\right)_{n=1}^{\infty}$ be a sequence of Banach spaces and let $V \subset\left(\sum_{n=1}^{\infty} Y_{n}\right)_{1}$ be a weakly compact set. Then for any $\varepsilon>0$ there exists an $n$ such that $\left\|P_{n}(v)\right\|<\varepsilon$ for every $v \in V$, where $P_{n}$ is the natural coordinate projection from $\left(\sum_{n=1}^{\infty} Y_{n}\right)_{1}$ onto the nth coordinate space.

Proof of the lemma. This is a standard and well-known "gliding hump" argument. If the conclusion fails for some $\varepsilon>0$, then $V$ contains a subsequence equivalent to the unit vector basis in $l_{1}$ (cf. [W, III.C.10]).

Theorem 2. Let $A \subset C(X)$ be a uniform algebra such that generalised peak points are dense in $X$ and $X$ has no isolated points. If $T: A \rightarrow A$ is a weakly compact operator then $\|I+T\|=1+\|T\|$, i.e., $A$ satisfies $(D E)$ for weakly compact operators.

Proof. Fix a small $\varepsilon>0$ and a function $f \in A,\|f\|=1$ such that $\|T f\|>$ $\|T\|-\varepsilon$. There exists an open set $U \subset X$ such that $|T f(x)-\|T\||<\varepsilon$ for $x \in$ $U$ (possibly we need to multiply the original $f$ by a scalar of absolute value 1 ). Let us fix a peak set $K \subset U$ such that $\left\|R_{K} T^{*}\right\|<\varepsilon$ (use Proposition 3) and find a function $h$ peaking on $K$. Using Mazur's Theorem (see [W, II.A.4]) and weak compactness of the operator $T$ we can find functions $\Psi_{k}=\sum_{j=N_{k}}^{N_{k+1}} \alpha_{j} h^{j}$ for $\left(N_{k}\right)$ a strictly increasing sequence of integers and $\sum_{j=N_{k}}^{N_{k+1}} \alpha_{j}=1=\sum_{j=N_{k}}^{N_{k+1}}\left|\alpha_{j}\right|$ such that the sequences $\left(T\left(\Psi_{k}\right)\right)_{k=1}^{\infty}$ and $\left(T\left(f \cdot \Psi_{k}\right)\right)_{k=1}^{\infty}$ are norm convergent. For any $\phi \in A^{*}$ we have

$$
\lim _{k \rightarrow \infty} \phi\left(T\left(\Psi_{k}\right)\right)=\lim _{k \rightarrow \infty}\left[T^{*}(\phi)\right]\left(\Psi_{k}\right)=R_{K} T^{*}(\phi)(1)
$$


and

$$
\lim _{k \rightarrow \infty} \phi\left(T\left(f \cdot \Psi_{k}\right)\right)=\lim _{k \rightarrow \infty}\left[T^{*}(\phi)\right]\left(f \cdot \Psi_{k}\right)=R_{K} T^{*}(\phi)(f) .
$$

From this we infer that for some integer $N$ and for all $k>N$ we have $\left\|T\left(\Psi_{k}\right)\right\|<\varepsilon$ and $\left\|T\left(f \cdot \Psi_{k}\right)\right\|<\varepsilon$. Let us look at functions $\left(1-\Psi_{k}\right)_{k=N}^{\infty}$. We would like to have $\left\|1-\Psi_{k}\right\|<1+\varepsilon$ for some $k>N$. If this does not happen, observe all functions $\left(1-\Psi_{k}\right)$ are zero on $K$ and that for every open set $V \supset K$ and any $\delta>0$ there is an integer $k$ such that $\left|1-\Psi_{k}(x)\right|<1+\delta$ for $x \in X \backslash V$. Using this we can inductively find (for any $M$ ) integers $k_{1}, k_{2}, \ldots, k_{M}$ such that sets $\left\{x \in X:\left|1-\Psi_{k_{j}}(x)\right|>1+\varepsilon / 2\right\}$ are disjoint. From this observation we see that

$$
\left\|1-\frac{1}{M} \sum_{j=1}^{M} \Psi_{k_{j}}\right\|_{\infty}=\left\|\frac{1}{M} \sum_{j=1}^{M}\left(1-\Psi_{k_{j}}\right)\right\|_{\infty}<1+\varepsilon
$$

if $M>4 / \varepsilon$. (This is a standard argument cf. [W, III.D.14].) If we put $\Psi=$ $\frac{1}{M} \sum_{j=1}^{M} \Psi_{k_{j}}$ we have

$$
\|1-\Psi\|_{\infty}<1+\varepsilon, \quad\|T(\Psi)\|_{\infty}<1+\varepsilon, \quad\|T(f \cdot \Psi)\|_{\infty}<1+\varepsilon .
$$

Observe that $\lim _{k \rightarrow \infty}\left\|(1-\Psi) f+\Psi_{k}\right\|_{\infty} \leq 1$ so we can find an integer $k$ such that for $\Phi=\Psi_{k}$ we have $\|(1-\Psi) f+\Phi\|<1+\varepsilon$ and $\|T \Phi\|<\varepsilon$. For $x \in K$ we have

$$
\begin{aligned}
\|(I+T)((1-\Psi) f+\Phi)\| & \geq|(1-\Psi(x)) f(x)+\Phi(x)+T((1-\Psi) f+\Phi)(x)| \\
& \geq|1+T(f)(x)|-(\|T(\Psi f)\|+\|T \Phi\|) \\
& \geq 1+\|T\|-3 \varepsilon .
\end{aligned}
$$

So we infer that $\|I+T\|=1+\|T\|$.

Let us discuss what uniform algebras satisfy the assumptions of Theorem 2. Clearly all $C(X)$ spaces for $X$ without isolated points do. When we consider an algebra $A$ on its Shilov boundary then the generalised peak points are dense. Thus the assumption about the density is not a real restriction. Also observe that if the Shilov boundary of an algebra $A$ has an isolated point then the characteristic function of this isolated point is an idempotent in $A$. Thus we have

Corollary 3. If a uniform algebra $A$ contains no nontrivial idempotents then it satisfies $(D E)$ for weakly compact operators.

Note that the assumptions of this corollary are satisfied by any (not onedimensional) closed subalgebra with 1 of $H_{\infty}(V)$ where $V$ is an open, connected subset of $\mathscr{C}^{n}$. Thus spaces like $H_{\infty}(V)$ and $A(V)$, where $V$ is a ball in $\mathscr{C}^{n}$ or a polydisc in $\mathscr{C}^{n}$, satisfy (DE) for weakly compact operators.

Since if $X^{*}$ satisfies (DE) for weakly compact operators then $X$ also does, we infer that $L_{1} / H_{1}$ satisfies (DE) for weakly compact operators (note that $\left.\left(L_{1} / H_{1}\right)^{*} \cong H_{\infty}\right)$. Also for $\mu$ an atomless measure, we have $L_{1}(\mu)^{*} \cong L_{\infty}(\mu) \cong$ $C(X)$ for $X$ without isolated points, so we infer (the well-known fact) that $L_{1}(\mu)$ for atomless $\mu$ satisfy (DE) for weakly compact operators. For more general operators on $L_{1}(\mu)$ the Daugavet Equation is proved in [PP]. For the history of this result the interested reader may consult the bibliography given in [A]. 
Now let $X$ be a complex Banach space, at least one-dimensional. Let us denote by $K$ the closed unit ball in $X^{*}$ equipped with $\sigma\left(X^{*}, X\right)$-topology. By $P(X)$ we denote the smallest closed subalgebra of $C(K)$ containing 1 and all functions $\hat{x}\left(x^{*}\right)=x^{*}(x)$ for $x \in X$. Clearly $P(X)$ is separable if $X$ is. It is a known result of Milne (cf. [M] or [W, III.I.4]) that $X$ is isometric to 1-complemented subspace of $P(X)$. Let $\Sigma$ be the Shilov boundary of $P(X)$ and let us consider $P(X)$ as a subalgebra of $C(\Sigma)$. We want to show that $\Sigma$ contains no isolated points. But it is clear that if a functional $x^{*} \in X^{*}, x^{*} \neq 0$, belongs to $\Sigma$, then also all functionals $e^{i t} x^{*}$ belong to $\Sigma$. Since the $\sigma\left(X^{*}, X\right)$ topology restricted to $\mathscr{C} \cdot x^{*} \subset X^{*}$ is equivalent to the usual topology, we see that no point of $\Sigma$ is isolated. (Note that $0 \in K$ cannot be a peak point.) Thus the theorem yields that for any Banach space $X$ the algebra $P(X)$ satisfies (DE) for weakly compact operators. So in particular, we have

Corollary 4. Every separable Banach space $X, X \neq\{0\}$ is isometric to a one complemented subspace of a separable Banach space satisfying (DE) for weakly compact operators.

\section{REFERENCES}

[A] Yuri Abramovich, New classes of spaces on which compact operators satisfy the Daugavet equation, J. Operator Theory (to appear).

[D] I. K. Daugavet, $A$ property of compact operators in the space $C$, Uspekhi Mat. Nauk 18 (1963), 157-158. (Russian)

[DU] J. Diestel and J. J. Uhl, Vector measures, Math. Surveys Monographs, vol. 15, Amer. Math. Soc., Providence, RI, 1977.

[G] T. W. Gamelin, Uniform algebras, Prentice Hall, Englewood Cliffs, NJ, 1969.

[M] H. Milne, Banach space properties of uniform algebras, Bull. London Math. Soc. 4 (1972), 323-326.

[PP] M. Plichko Anatolii and M. Popov Mikhail, Symmetric function spaces on atomless probability spaces, Dissertationes Math. 306 (1990).

[W] P. Wojtaszczyk, Banach spaces for analysts, Cambridge Stud. Adv. Math., vol. 25, Cambridge Univ. Press, Cambridge, 1991.

Institut of Mathematics, Warsaw University, 00-913 Warszawa 59 ul. Banacha 2, POLAND

E-mail address: PRZEMEK@PLEARN.BITNET 